\title{
A THEORY OF SOFTWARE REUSABILITY
}

\author{
M.C. GAUDEL ${ }^{1}$ and Th. MOINEAU ${ }^{1,2}$
}

(1) Laboratoire de Recherche en Informatique

Unité associée au CNRS UA 410

Bât. 490, Université Paris-Sud

91405 ORSAY CEDEX

FRANCE

\author{
(2) SEMA-METRA
}

16 Rue Barbès

92126 MONTROUGE

FRANCE

\begin{abstract}
Software reusability is a topic of first practical importance. Most of the current approaches are based on empirical methods and there is no general approach to this problem. This paper suggests a definition for software reusability based on algebraic specifications and modularity. This criterion is not completely constructive, but it provides a guideline to find out reusable software components and prove their reuse. Moreover, we state how to exploit reusability in hierarchical specifications.
\end{abstract}

Key-words : reuse, abstract data types, formal specifications, modularity, PLUSS.

\section{Introduction}

Software reusability is a topic of first practical importance. Most of the current approaches are based on empirical methods such as key words or descriptions in natural language. For some specific fields there exist good libraries of software components, and the description of the component is given in the terminology of the application area (mathematics, management, ...). However, there is no general approach to this problem.

To reuse a piece of software is only possible if what this piece of software does is precisely stated. It means that a specification of this software component is available. In this paper, we consider the case of software components which are formally specifed using algebraic specifications. We deal with the following problem : given a specification $S P^{\prime}$ to be implemented, and a specification $S P$ of an already implemented software component, is this component reusable for the implementation of $S P^{\prime}$ ? Thus we are not considering reusability of software design, or reusability of specification (which are also important problems) but reusability of code. A software component library should, at least, contain couples of the form <formal specification, piece of code>. Our claim is that the use of formal and structured specifications is fundamental for reusability. We define rigorously, in the case of algebraic structured specifications, the relations "is reusable for" and "is efficiently reusable for" between two specifications, the first one being already implemented. Moreover, it turns out that these definitions fit well with the primitives of our specification language.

We consider partial algebraic data types [GH 78,BW 82] and hierarchical specifications [WPPDB 83]. The specification language we use for our examples is PLUSS [Gau 85,Bid 87] which 
is based on the ASL primitives [Wir 83]. The first part of the paper is a short presentation of these basic concepts. In part 2 we define, following [Bid 87], the semantics of the use of predefined specifications. Part 3 is an informal introduction of our definitions of reusability. Part 4 states precisely these definitions. Part 5 explores the relationship between reusability and hierarchy : several theorems are given which show that our definitions are compatible both with the classical definitions of hierarchical specifications and with the practical aspects of software reusability.

Thus this paper suggests a criterion for software reusability which is theoretically founded. This criterion is not completely constructive, but it provides a guideline to find out reusable software components and prove their reuse. Moreover, the theorems of part 5 state how to exploit reusability in hierarchical specifications.

\section{Basic definitions}

A signature $\Sigma=(S, F)$ consists of a set $S$ of sort names and a set $F$ of operation symbols, for each of which a profile $s_{1} \ldots s_{n} \longrightarrow s_{n+1}$ with $s_{i} \in S$ is given.

A $\Sigma$-algebra $A$ is a family $\left(s^{A}\right)_{s \in S}$ of carrier sets together with a family of partial functions $\left(f^{A}\right)_{f \in F}$, such that the profiles of the operation names $f \in F$ coincide with the profiles of the functions $f^{A}$.

A (total) $\Sigma$-morphism $\phi$ from $A$ to $B$ is a family $\left(\phi_{s}\right)_{s \in S}$ of (total) applications $\phi_{s}: s^{A} \longrightarrow s^{B}$, such that for all operation name $\left(f: s_{1}, \ldots s_{n} \rightarrow s\right) \in F$ and all objects $a_{1} \in s_{1}^{A}, \ldots, a_{n} \in s_{n}^{A}$ the following holds : if $f^{A}\left(a_{1}, \ldots, a_{n}\right)$ is defined then $\phi_{s}\left(f^{A}\left(a_{1}, \ldots, a_{n}\right)\right)=f^{B}\left(\phi_{s_{1}}\left(a_{1}\right), \ldots, \phi_{s_{n}}\left(a_{n}\right)\right)$. Two $\Sigma$-algebra $A$ and $B$ are isomorphic, written $A \simeq B$, iff there is an isomorphism (bijective $\Sigma$-morphism) between them.

$T_{\Sigma}$ is the well-known term-algebra. The interpretation $t^{A}$ of a term $t$ in a $\Sigma$-algebra $A$ is specified by :

- if $t=c \in F$, then $t^{A}={ }_{d e f} c^{A}$.

- if $t=f t_{1} \ldots t_{n}$, then $t^{A}={ }_{\text {def }} f^{A}\left(t_{1}^{A}, \ldots, t_{n}^{A}\right)$ provided that all the interpretations $t_{i}^{A}$ and $f\left(t_{1}^{A}, \ldots, t_{n}^{A}\right)$ are defined; otherwise $t^{A}$ is undefined.

$T_{\Sigma U V}$ is the free $\Sigma$-algebra of terms with variables in $V$.

$P A L G(\Sigma)$ is the category of the partial $\Sigma$-algebras with $\Sigma$-morphisms, $P G E N(\Sigma)$ is the category of finitely generated $\Sigma$-algebras (i.e. all elements of the carrier sets can be obtained by the interpretation $t^{A}$ of a term $t \in T_{\Sigma}$ ).

A specification $S P=(\Sigma, E)$ consists of a signature $\Sigma$ and a set $E$ of positive conditional axioms on $\Sigma: \Phi_{1} \wedge \ldots \wedge \Phi_{n} \Longrightarrow \Phi_{n+1}$, where $\Phi_{i}$ are either equations $\left(t_{1}=t_{2}\right)$ or definedness predicates $D(t)$ where $t_{1}, t_{2}$ and $t$ are terms of $T_{\Sigma u V}$ (a $\Sigma$-algebra $A$ satisfies $D(t)$ iff $t^{A}$ is defined for all assignments of its variables).

$P A L G(S P)$ is the category of all the models of $S P$ (the $\Sigma$-algebras satisfying all axioms of E). $P G E N(S P)$ is those of finitely generated models. $T_{S P}$ is the initial model of $P A L G(S P)$ and $P G E N(S P)$. If $A \in P A L G(S P), I_{A}$ is the unique $\Sigma$-morphism $I_{A}: T_{S P} \longrightarrow A$.

We use the abbreviation $S P_{0} \subseteq S P$ for $\Sigma_{0} \subseteq \Sigma\left(S_{0} \subseteq S\right.$ and $\left.F_{0} \subseteq F\right)$ and $E_{0} \subseteq E$. We write $S P=S P_{0} \cup \Delta S P$ for $\Sigma=\Sigma_{0} \cup \Delta \Sigma\left(S=S_{0} \cup \Delta S\right.$ and $\left.F=F_{0} \cup \Delta F\right)$ and $E=E_{0} \cup \Delta E . \Delta S P$ is called an enrichment. Note that these definitions are purely syntactic (union of presentations).

If $S P_{0} \subseteq S P$, we note $U: P A L G(\Sigma) \rightarrow P A L G\left(\Sigma_{0}\right)$ the forgetful functor defined by : $s^{U(A)}=s^{A}$ for $s \in S_{0}$ and $f^{V(A)}=f^{A}$ for $f \in F_{0} . U(A)$ corresponds to the $\Sigma_{0 \text {-reduct of } A \text { as }}$ defined in [WPPDB 83]. 
As mentioned in the introduction, we will deal with hierarchical specifications denoted by $S P=S P_{0}+\triangle S P$ (this corresponds to the ENRICH construct of ASL).

\section{Hierarchical models, implementations, realizations}

\subsection{Hierarchical models}

As a preliminary to any study of reusability, it is necessary to define what is a correct implementation of a specification. This definition is dependent on the semantics considered for the specifications, i.e. the models associated with the specification. There are several approaches :

1. the models are those isomorphic to $T_{S P}$; such a semantics is called initial semantics.

2. the models are those isomorphic to the terminal algebra; such a semantics is called terminal semantics.

3. the models are all the models in $P A L G(S P)$, such a semantics is called "loose" semantics.

4. a loose semantics may consider only finitely generated models, those in $P G E N(S P)$ [WPPDB 83].

Loose semantics makes it possible to give a simple definition of implementations [SW 82] : an algebra is an implementation of a specification SP, if, via some forgetting, restriction and identification, it is a model of SP.

However, loose semantics without hierarchy introduces trivial algebras among the models : the solution is to start from some basic specifications (such as booleans, naturals) with initial semantics, and to consider enrichments of specifications with hierarchical constraints.

Most of the time, it is convenient to consider finitely generated models : all the values are denotable by a term and thus are computable in a finite number of steps. Non finitely generated models are sometimes useful, but as we are concerned with reusability, it seems sound to choose finitely generated models : it means that the specification of a software component is supposed to mention all the functions of this components.

Thus we consider that the semantics of a specification SP is a subclass of $P G E N(S P)$ noted $H M O D(S P)$ of hierarchical finitely generated models, where the hierarchical constraints ensure that any model (implementation) of $S P=S P_{0}+\triangle S P$ restricts into a model of $S P_{0}$. More precisely the forgetful functor from $S P$ into $S P_{0}$ applied to a hierarchical model of $S P$ gives a hierarchical model of $S P_{0}$.

Definition 2.1 The class of the hierarchical models of a specification is :

- $H M O D\left(S P_{0}\right)=\left\{T_{S P_{0}}\right\}$ if $S P_{0}$ is a basic specification $\left(\Sigma_{0}, E_{0}\right)$.

- $H M O D(S P)=\left\{A \in P G E N(S P) \mid U(A) \in H M O D\left(S P_{0}\right)\right\}$ if $S P$ is a hierarchical specification: $S P=S P_{0}+\triangle S P\left(U\right.$ is the forgetful functor from $P A L G(\Sigma)$ into $\left.P A L G\left(\Sigma_{0}\right)\right)$.

$H M O D(S P)$ is a full sub-category of $P G E N(S P)$. The existence of initial or terminal model is not ensured ( $T_{S P}$ is not always a hierarchical model [Ber 87]).

Of course, the class of models of a specification depends of its hierarchy; for instance : $H M O D\left(\left(S P_{0}+\Delta S P_{1}\right)+\triangle S P_{2}\right) \subseteq H M O D\left(S P_{0}+\left(\Delta S P_{1} \cup \Delta S P_{2}\right)\right)$

But the reverse is not always true. This will turn out to be quite important for reusability aspects.

We do not restrict the class of models to minimally defined ones [BW 82]. It means that an operation can be implemented in such a way that it is more defined than what is specified. This seems interesting in the framework of reusability. 


\subsection{Realization}

As stated in introduction, we consider couples <specification, program>, where the program is correct with respect to the specification. In the algebraic specification framework [EKMP 80,5W 82,BBC 86] it means that the program is a model of the specification modulo some forgettings, restrictions and identifications.

However, as soon as software is modular, we are concerned mainly with pieces of software which use other pieces of software. For instance, a module implementing a specification of the sets of integers includes some code for the operations insert and is-a-member, and uses some already existing implementation of the operations on integers. Thus, given a structured specification $S P=S P_{0}+\triangle S P$ we call a "realization" of $\triangle S P$ a piece of software such that when coupling it with a hierarchical model (implementation) of $S P_{0}$, one gets a hierarchical model of $S P$. In order to be reusable, a realization of $\triangle S P$ must accept any model of $S P_{0}$ : no assumptions can be made on the implementation of $S P_{0}$. This property is not only important for reusability, but also in large software projects where several programmers are working concurrently on various modules.

These considerations lead to the following definition :

Definition 2.2 A realization of enrichment $\triangle S P$ of $S P_{0}$ is a functor $\triangle$ from $H M O D\left(S P_{0}\right)$ into $H M O D\left(S P_{0}+\triangle S P\right)$ such that : $\forall A_{0} \in H M O D\left(S P_{0}\right), U\left(\Delta\left(A_{0}\right)\right) \simeq A_{0}$.

The class of realizations of $\triangle S P$ on the top of $S P_{0}$ is noted $R E A L_{S P_{i}}(\triangle S P)$.

As stated above, this functor must be conservative. For instance, if $S P_{0}$ is an implementation of the integers by 8 bits strings, $\Delta$ will provide an implementation of $S P$ where integers are implemented by 8 bits strings, not by 16 bits strings : there is no recoding of the integers by the realization of, for instance, the sets of integers.

Composition of realizations works well :

Theorem 2.1 Let $S P_{1}=S P_{0}+\Delta S P_{1}, S P_{2}=S P_{1}+\Delta S P_{2}$ and $\Delta S P_{3}=\Delta S P_{1} \cup \Delta S P_{2}$. If $\Delta_{1} \in R E A L_{S P_{i}}\left(\triangle S P_{1}\right)$ and $\Delta_{2} \in R E A L_{S P_{1}}\left(\triangle S P_{2}\right)$, then $\Delta_{2} \circ \Delta_{1} \in R E A L_{S P_{4}}\left(\triangle S P_{3}\right)$.

Proof : The forgetful functor $U_{3}$ from $A L G\left(S P_{3}\right)$ into $A L G\left(S P_{0}\right)$ is equal to $U_{1} \circ U_{2}$ where $U_{1}$ et $U_{2}$ are the forgetful functors from $A L G\left(S P_{1}\right)$ into $A L G\left(S P_{0}\right)$ and from $A L G\left(S P_{2}\right)$ into $A L G\left(S P_{1}\right)$. Thus $\forall A_{0} \in H M O D\left(S P_{0}\right): U_{3}\left(\Delta_{2} \circ \Delta_{1}\left(A_{0}\right)\right)=U_{1} \circ\left(U_{2} \circ \Delta_{2}\right) \circ \Delta_{1}\left(A_{0}\right) \simeq$ $U_{1} \circ \Delta_{1}\left(A_{0}\right) \simeq A_{0}$.

It is important to note that there does not always exist a realization for a $\triangle S P$. If $\triangle S P$ removes some models from $H M O D\left(S P_{0}\right)$, assuming for instance some implementation choices of $S P_{0}$, there exist no realization. However $S P=S P_{0}+\triangle S P$ may have hierarchical models, thus global implementations. The existence of a realization means that the implementation choices in $\triangle S P$ and in $S P_{0}$ are completely independent.

\section{What is reusability ?}

\subsection{Intuitive introduction}

This part is an intuitive introduction to the formal definitions which are given in part 4.

Let us consider an example : suppose we have in a software components library a program corresponding to the specification INT of figure 1; we want to develop a program corresponding 


\begin{tabular}{|c|}
\hline SPEC : NAT \\
USE : BOOL \\
SORT : Nat \\
OP : $0, \mathrm{~s}$ \\
,$+ *$, fact, $\leq$ \\
AXIOMS : \\
$0+\mathrm{y}=\mathrm{y}$ \\
$\mathrm{s}(\mathrm{x})+\mathrm{y}=\mathrm{s}(\mathrm{x}+\mathrm{y})$ \\
$0 * \mathrm{y}=0$ \\
$\mathrm{~s}(\mathrm{x}) * \mathrm{y}=(\mathrm{x} * \mathrm{y})+\mathrm{y}$ \\
fact $(0)=\mathrm{s}(0)$ \\
fact $(\mathrm{s}(\mathrm{x}))=\mathrm{s}(\mathrm{x}) *$ fact $(\mathrm{x})$ \\
$0 \leq 0=\mathrm{true}$ \\
$\mathrm{s}(\mathrm{x}) \leq 0=$ false \\
$0 \leq \mathrm{s}(\mathrm{x})=$ true \\
$\mathrm{s}(\mathrm{x}) \leq \mathrm{s}(\mathrm{y})=\mathrm{x} \leq \mathrm{y}$ \\
WHERE : \\
$\mathrm{x}, \mathrm{y}:$ Nat \\
\hline
\end{tabular}

\section{SPEC : INT}

USE : BOOL

SORT : Int

OP : zero, succ, pred $+,-, \leq$

AXIOMS :

$$
\begin{aligned}
& \operatorname{succ}(\operatorname{pred}(x))=x \\
& \operatorname{pred}(\operatorname{succ}(x))=x \\
& \mathrm{x}+\text { zero }=\mathrm{x} \\
& x+\operatorname{succ}(y)=\operatorname{succ}(x+y) \\
& x+\operatorname{pred}(y)=\operatorname{pred}(x+y) \\
& \mathrm{x}-\text { zero }=\mathrm{x} \\
& x-\operatorname{succ}(y)=\operatorname{pred}(x-y) \\
& x-\operatorname{pred}(y)=\operatorname{succ}(x-y) \\
& \mathrm{x} \leq \mathrm{x}=\text { true } \\
& \text { zero } \leq \operatorname{pred}(\text { zero })=\text { false } \\
& \text { zero } \leq \mathrm{x}=\text { true } \\
& \Longrightarrow \text { zero } \leq \operatorname{succ}(x)=\text { true } \\
& \text { zero } \leq \mathrm{x}=\text { false } \\
& \Longrightarrow \text { zero } \leq \operatorname{pred}(x)=\text { false } \\
& \operatorname{succ}(\mathrm{x}) \leq \mathrm{y}=\mathrm{x} \leq \operatorname{pred}(\mathrm{y}) \\
& \operatorname{pred}(x) \leq y=x \leq \operatorname{succ}(y)
\end{aligned}
$$

WHERE :

$x, y:$ Int

Figure 1: NAT and INT specifications

to the specification NAT of figure 1. Following our intuition and our experience of programming, it is obvious that the code of INT is reusable for implementing NAT. More precisely, BOOL, Nat, $0, s$ and $\leq$ can be implemented by reusing INT.

In this example, we notice that:

- a renaming is needed between INT and NAT;

- the axioms of INT and NAT are different;

- INT provides functions which are not required by NAT;

- some functions required by NAT are missing in INT.

These remarks lead to the scheme of figure 2 which shows that reusability of $S P$ for $S P^{\prime}$ w.r.t. a subsignature $\Sigma_{r}^{\prime}$ of $\Sigma^{\prime}$ implies the existence of two specifications $S P_{1}$ and $S P_{1}^{\prime}$ such that:

1. $S P_{1}$ is an extension of $S P$ : the missing functions (and possibly some hidden functions) are added to $S P: S P_{1}=S P+\triangle S P_{1}$. Practically speaking, a realization of $\Delta S P_{1}$ must be developed, since the reused program is only known by its specification $S P$.

2. $S P_{1}^{\prime}$ is an enrichment of $S P^{\prime}$ which is equivalent, modulo a renaming, to $S P_{1}$. This equivalence states the validity of the reuse of $S P$ for $S P^{\prime}$ and makes it possible to have different axioms in $S P$ and $S P^{\prime}$, as in example of figure 1 . A more (and too) restrictive definition of reusability could consider only syntactic renaming from $S P_{1}$ to $S P_{1}^{\prime}$.

3. Any model of $S P_{1}^{\prime}$ can be restricted via the forgetful functor to a model of $S P^{\prime}$ : unnecessary 


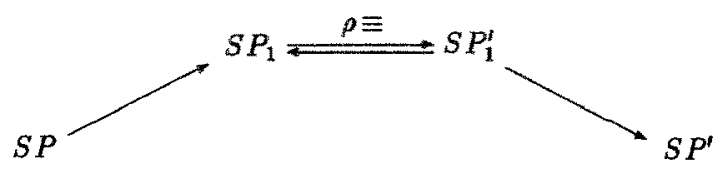

Figure 2: Scheme of reuse.

functions of $S P$, and hidden functions are forgotten :

$$
\forall A_{1}^{\prime} \in H M O D\left(S P_{1}^{\prime}\right), U^{\prime}\left(A_{1}^{\prime}\right) \in H M O D\left(S P^{\prime}\right)
$$

4. In order to ensure that sorts and operations of $\Sigma_{r}^{\prime}$ are actually reused from $S P, \Sigma_{r}^{\prime}$ must be a renaming of a subsignature of $\Sigma$.

However condition 3 is too restrictive in some cases : if we consider the example of figure 1 , implementing NAT by reuse of INT, the result of the forgetful functor is not a (hierarchical) finitely generated model of NAT, since the negative integers are kept. Thus we need a "stronger" forgetful functor than the classical one : by the way, it is a forget-restrict functor in the sense of [EKMP 80,SW 82]. Other problems arise if some functions are more defined in $S P$ than in $S P^{\prime}$. Part 4 gives the corresponding definitions.

\subsection{Reusability vs abstract implementation}

Reuse is a special, simpler case of abstract implementation of algebraic data types [EKMP $80, \mathrm{SW} 82, \mathrm{BBC} 86$ ]. In this paper we are concerned by direct reuse. It means that the "abstraction functions" of [BBC 86] or the "copy functions" of [EKMP 80] are just identities, and that the "identify function" is no more necessary to get a model of $S P^{\prime}$.

\section{Reusability : definitions}

As mentioned above, we have a reusability definition in three steps : extension, equivalence modulo a renaming, and forget-restricting. We study each of them successively.

\subsection{Extension}

The extension phase consists in giving an enrichment $\Delta S P_{1}$ on the top of $S P$, which must be realizable :

Definition 4.1 $\triangle S P_{1}$ is realizable on the top of $S P_{0}$ iff $R E A L_{S P_{0}}(\triangle S P) \neq \emptyset$.

This property is noted $S P_{0} \longrightarrow S P$ or $S P=S P_{0} \oplus \triangle S P$.

\section{Remark.}

$R E A L_{S P_{0}}(\triangle S P)$ is the semantics of the USE construct of the PLUSS specification language [Bid 87]. PLUSS makes a distinction between the USE construct, which corresponds to the modular structure of the software, and the ENRICH construct which expresses the incremental development of specifications and which has the same semantics as in ASL. 
$S P=S P_{0} \oplus \triangle S P$ corresponds to the following construct in PLUSS :

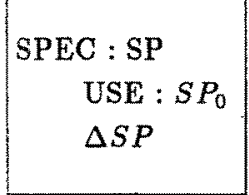

Realizability is a transitive relation :

Theorem 4.1 If $S P_{1}=S P_{0} \oplus \Delta S P_{1}$ and $S P_{2}=S P_{1} \oplus \Delta S P_{2}$ then $S P_{3}=S P_{0} \oplus\left(\Delta S P_{1} \cup \Delta S P_{2}\right)$.

Proof : Let $\Delta S P_{3}=\Delta S P_{1} \cup \triangle S P_{2}$. One can take $\Delta_{1} \in R E A L_{S P_{0}}\left(\triangle S P_{1}\right)$ and

$\Delta_{2} \in R E A L_{S P_{1}}\left(\triangle S P_{2}\right)$. From theorem 2.1, $\Delta_{2} \circ \Delta_{1} \in R E A L_{S P_{0}}\left(\triangle S P_{3}\right)$, and thus $R E A L_{S P_{0}}\left(\triangle S P_{3}\right) \neq \emptyset$.

Note that $H M O D\left(S P_{3}\right)$ is generally different from $H M O D\left(S P_{2}\right) . S P_{2}$ and $S P_{3}$ are equal if they are considered as non hierarchical specifications, but are different as hierarchical ones.

The definitions above does not cope with parameterization : $\Delta$ may perfectly take into account some properties of the specification $S P_{0}$. It must not take into account properties of the implementation of $S P_{0}$. Parameterized specifications and generic modules introduce additional difficulties and are not considered in this paper.

\subsection{Equivalence of hierarchical specifications modulo a renaming}

A renaming between two signatures $\Sigma_{1}$ and $\Sigma_{2}$ is a signature isomorphism $\rho: \Sigma_{1} \rightarrow \Sigma_{2}$ [EM 85]. Some authors define renamings as injective signature morphisms [Pro 82]. Since we are interested in equivalence of specifications, we consider bijective renamings. Signature renamings easily extend into term renamings, algebra renamings, and (hierarchical) specification renamings.

The classical notion of specification equivalence is that $S P$ and $S P^{\prime}$ are equivalent iff $\Sigma=\Sigma^{\prime}$ and $T_{S P} \simeq T_{S P^{i}}$. For hierarchical specifications, the definition is :

Definition 4.2 : hierarchical equivalence.

Two basic specifications $S P_{0}$ and $S P_{0}^{\prime}$ are hierarchically equivalent iff $\Sigma_{0}=\Sigma_{0}^{\prime}$ and $T_{S P_{i}} \simeq T_{S P_{n}^{t}}$.

Two hierarchical specifications $S P=S P_{0}+\triangle S P$ and $S P^{\prime}=S P_{0}^{\prime}+\Delta S P^{\prime}$ are hierarchically equivalent iff :

- $S P_{0}$ and $S P_{0}^{\prime}$ are hierarchically equivalent

$-\Delta \Sigma=\Delta \Sigma^{\prime}$.

- $H M O D(S P)=H M O D\left(S P^{\prime}\right)$.

Hierarchical equivalence is denoted by $S P \equiv S P^{\prime}$. It means that two hierarchical specifications are hierarchically equivalent iff they have the same (unfolded) signature, the same hierarchy, the same hierarchical models. Only the axioms can differ.

Proposition 4.2 Let $S P=S P_{0}+\Delta S P$ and $S P^{\prime}=S P_{0}^{\prime}+\Delta S P^{\prime}$. If $S P_{0} \equiv S P_{0}^{\prime}, \Delta \Sigma=\Delta \Sigma^{\prime}$ and $T_{S P_{0}} \simeq T_{S P_{n}^{\prime}}$, then $H M O D\left(S P_{0}+\Delta S P\right)=H M O D\left(S P_{0}^{\prime}+\Delta S P^{\prime}\right)$ and thus $S P \equiv S P^{\prime}$.

Proof : by induction on the hierarchy.

From the definitions above, it comes :

Definition 4.3 Two hierarchical specifications $S P$ and $S P^{\prime}$ are hierarchically equivalent modulo a renaming $\rho\left(S P \rho \equiv S P^{\prime}\right)$ iff $\rho(S P) \equiv S P^{\prime}$. 


\subsection{Forget-restrict functor}

As noted above, we need a forget-restrict functor in order to get finitely generated models of $S P^{\prime}$ (see fig. 2).

Definition 4.4 Let $S P^{\prime} \subseteq S P_{1}^{\prime}$ two specifications.

The forget-restrict functor $V: P A L G\left(S P_{1}^{\prime}\right) \longrightarrow P G E N\left(S P^{\prime}\right)$ is defined by :

- $\forall A \in P A L G\left(S P_{1}^{\prime}\right)$,

$-\forall s \in S^{\prime}, s^{V(A)}=\left[I_{U(A)}\right]_{0}\left(s^{T_{S P^{\prime}}}\right)$.

$-\forall\left(f: s_{1} \ldots s_{n} \longrightarrow s\right) \in F^{\prime}$,

$$
f^{V(A)}\left(a_{1}, \ldots a_{n}\right)= \begin{cases}f^{U(A)}\left(a_{1}, \ldots, a_{n}\right) & \text { if } f^{V(A)}\left(a_{1}, \ldots, a_{n}\right) \in s^{V(A)} \\ \text { undefined } & \text { otherwise }\end{cases}
$$

- $\forall \phi: A \longrightarrow B, V(\phi)$ is the restriction of $U(\phi)$ to $V(A)$.

As defined above, $I_{U(A)}$ is the unique morphism from $T_{S P^{\prime}}$ into $U(A)$.

$\mathrm{V}(\mathrm{A})$ is the finitely generated part of $U(A)$ with respect to $\Sigma^{\prime}\left(s^{V(A)}=\left\{a \in s^{A} \mid \exists t \in T_{S p^{\prime}}, a=\right.\right.$ $\left.\left.t^{A}\right\}\right)$. By the way, the sorts of $S P^{\prime}$ are subsorts of those of $S P_{1}^{\prime}$ [FGJM 85]. It's easy to prove that $V$ is a functor from $P A L G\left(S P_{1}^{\prime}\right)$ into $P G E N\left(S P^{\prime}\right)$, and that composition of forget-restrict functors works well : if $V_{2}$ is the forget-restrict functor from $S P_{2}$ to $S P_{1}$ and $V_{1}$ is the forget-restrict functor from $S P_{1}$ to $S P_{0}$ then $V_{1} \circ V_{2}$ is the forget-restrict functor from $S P_{2}$ to $S P_{0}$.

Besides, by definition, the forget-restrict functor restricts the domain of functions in such a way that only finitely generated objects can be got as result.

\subsection{Reusability}

We now put together the three steps and define reusability.

Definition 4.5 $S P$ is reusable for $S P^{\prime}$ w.r.t. a signature $\Sigma_{r}^{\prime}$ modulo a renaming $\rho$ $\left(S P \rho \hookrightarrow S P^{\prime}\left[\Sigma_{r}^{\prime}\right]\right)$ iff there exist two specifications $S P_{1}$ and $S P_{1}^{\prime}$ such that :

[R1] $S P_{1}=S P \oplus \Delta S P_{1}$.

[R2] $S P_{1} \rho \equiv S P_{1}^{\prime}$.

[R3] $S P^{\prime} \subseteq S P_{1}^{\prime}$.

[R4a] $\forall A_{1}^{\prime} \in H M O D\left(S P_{1}^{\prime}\right), V^{\prime}\left(A_{1}^{\prime}\right) \in H M O D\left(S P^{\prime}\right)$.

[R5] $\Sigma_{f}^{\prime} \subseteq \Sigma^{\prime}$ and $\rho^{-1}\left(\Sigma_{r}^{\prime}\right) \subseteq \Sigma$.

where $V^{\prime}$ is the forget-restrict functor from $P A L G\left(S P_{1}^{\prime}\right)$ into $P G E N\left(S P^{\prime}\right)$.

Note that in $A_{1}^{\prime}$, some functions can be more defined than what is required by $S P^{\prime}$. It does not matter since $H M O D\left(S P^{\prime}\right)$ is not limited to minimally defined models (as defined in [BW 82]).

Definition 4.6 $S P$ is efficiently reusable for $S P^{\prime}$ w.r.t. a signature $\Sigma_{r}^{\prime}$ modulo a renaming $\rho\left(S P \rho \leadsto S P^{\prime}\left[\Sigma_{r}^{t}\right]\right)$ iff there exist two specifications $S P_{1}$ and $S P_{1}^{\prime}$ such that :

[R1] $S P_{1}=S P \oplus \Delta S P_{1}$.

[R2) $S P_{1} \rho \equiv S P_{1}^{\prime}$. 
[R3] $S P^{\prime} \subseteq S P_{1}^{\prime}$.

$[\mathrm{R} 4 \mathrm{~b}] \forall A_{1}^{\prime} \in H M O D\left(S P_{1}^{\prime}\right), U\left(A_{1}^{\prime}\right) \in H M O D\left(S P^{\prime}\right)$.

[R5] $\Sigma_{r}^{\prime} \subseteq \Sigma^{\prime}$ and $\rho^{-1}\left(\Sigma_{r}^{\prime}\right) \subseteq \Sigma$.

where $U^{\prime}$ is the forgetful functor from $P A L G\left(S P_{1}^{\prime}\right)$ into $P A L G\left(S P^{\prime}\right)$.

The intuitive notion behind efficient reusability is that the carriers of $S P$ are finitely generated with respect to $S P^{\prime}$ : there are no useless values.

At the practical level, our definition of reusability in three steps will result in specifications of the form :

\section{SPEC : $X$ FORGET $\ldots$ \\ (USE : SP \\ $\left.\triangle S P_{1}\right)$ RENAMING $\ldots$ INTO $\ldots$ END $X$}

Note that $X$ is an abstract implementation of $S P^{\prime}: H M O D(X) \subseteq H M O D\left(S P^{\prime}\right)$.

The second step (equivalence modulo a renaming) can be skipped : $S P_{1}$ and $S P_{1}^{\prime}$ can be embedded in an unique but more complex specification $S P_{2}$ :

Theorem 4.3 $S P$ is reusable (resp. efficiently reusable) for $S P^{\prime}$ w.r.t. $\Sigma_{r}^{\prime}$ modulo a renaming $\rho$ iff there exists a specification $S P_{2}$ such that :

[1] $S P_{2}=S P \oplus \triangle S P_{2}$.

[2] $S P^{\prime} \subseteq \rho\left(S P_{2}\right)$.

[3] $\forall A_{2} \in H M O D\left(S P_{2}\right), V^{\prime}\left(\rho\left(A_{2}\right)\right) \in H M O D\left(S P^{\prime}\right)\left(\right.$ resp. $\left.U^{\prime}\left(\rho\left(A_{2}\right)\right) \in H M O D\left(S P^{\prime}\right)\right)$.

[4] $\Sigma_{r}^{\prime} \subseteq \Sigma^{\prime}$ and $\rho^{-1}\left(\Sigma_{r}^{\prime}\right) \subseteq \Sigma$.

Proof : $\Longleftarrow$ Take $S P_{1}=S P_{2}$ and $S P_{1}^{\prime}=\rho\left(S P_{2}\right)$.

$\Longrightarrow$ Take $S P_{2}=\left(\Sigma_{1}, E_{1} \cup \rho^{-1}\left(E_{1}^{\prime}\right)\right)$. Since $S P_{1} \rho \equiv S P_{1}^{\prime}$ the axioms of $S P_{1}^{\prime}$ (renamed by $\left.\rho^{-1}\right)$

do not change the hierarchical models of $S P_{1}$.

This approach is less close to reality, but easier to deal with theoretically. Consequently, in the rest of this paper we will consider reuses in two steps. Besides, we ignore renaming, since it is only a matter of syntactic sugar. However, the results still hold in the case of reuse with a renaming. Reusability and efficient reusability without renaming are noted $\hookrightarrow$ and $\leadsto$. We will denote a specific reuse of $S P$ for $S P^{\prime}$ by the scheme $: S P \longrightarrow S P_{1} \searrow^{V} S P^{\prime}\left[\Sigma_{r}^{\prime}\right]$, and an efficient reuse by $S P \longrightarrow S P_{1} \searrow^{U} S P^{\prime}\left[\Sigma_{r}^{\prime}\right]$.

\section{Properties.}

- if $S P$ is efficiently reusable for $S P^{\prime}$ w.r.t. $\Sigma_{r}^{\prime}$ then $S P$ is reusable for $S P^{\prime}$ w.r.t. $\Sigma_{r}^{\prime}$.

- if $S P \longrightarrow S P^{\prime}$ then $S P$ is efficiently reusable for $S P^{\prime}$ w.r.t. $\Sigma$. 


\section{Example.}

The following example illustrates these definitions. We consider four specifications and look at the relations of reusability between them.
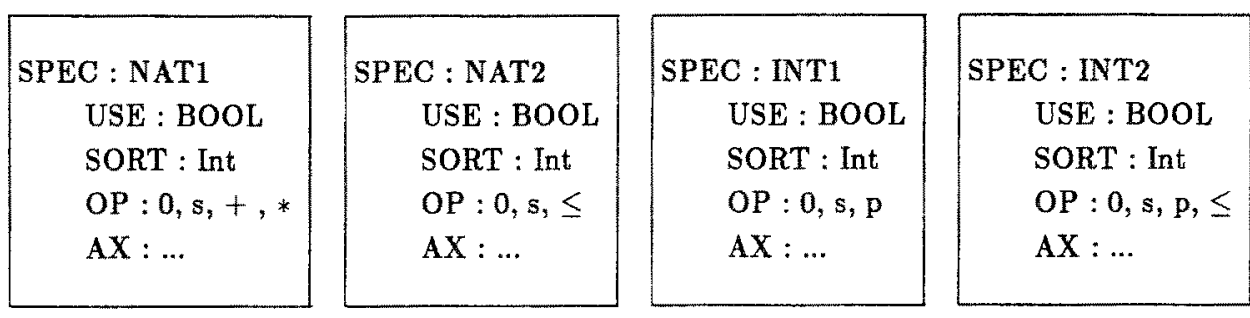

The axioms are the classical ones and are omitted. The hierarchical models are :

- $H M O D(\mathrm{NAT} 1)=\{N\} \cup\{Z / n Z \mid n \in N\}$

- HMOD(NAT2) $=\{N\}$

- $H M O D(\mathrm{INT} 1)=\{Z\} \cup\{Z / n Z \mid n \in N\}$

- $H M O D($ INT2 $)=\{Z\}$

Thus :

- NAT2 is efficiently reusable for NAT1 w.r.t. $\Sigma_{B O O L} \cup\langle\{\ln t\},\{0, s\}\rangle$.

- INT1 is reusable for NAT1 w.r.t. $\Sigma_{B O O L} \cup\langle\{$ Int $\},\{0, s\}\rangle$.

- INT2 is reusable for NAT2 w.r.t. $\Sigma_{B O O L} \cup\{\{$ Int $\},\{0, s, \leq\}\rangle$.

But :

- NAT1 is not reusable for NAT2 w.r.t. $\Sigma_{B O o L} \cup\langle\{I n t\},\{0, s\}\rangle$. Since $Z / 2 Z$ is a NAT1 model for which there is no extension into $N$, and $N$ is the only model of NAT2.

- NAT1 is not reusable for INT1 w.r.t. $\Sigma_{B O O L} \cup\langle\{$ Int $\},\{0, s\}\}$.

- NAT2 is not reusable for INT2 w.r.t. $\Sigma_{B O O L} \cup\langle\{$ Int $\},\{0, s, \leq\}\rangle$.

The reusability and efficient reusability relations are not symmetric (see NAT1 and INT1 above). This is not surprising as soon as we want integers to be reusable for the naturals but not the reverse. The transitivity of these relations is discussed below.

\section{Reusability, reuse and hierarchy}

Our formal definition of reusability is applicable only if it is compatible with the primitives of most of the specification languages. This part of the paper discusses the relationship between reusability, reuse and hierarchy.

\subsection{What about primitives specifications?}

The first result we give is negative :

Fact 5.1 There exist specifications $S P$ and $S P^{i}$ such that $S P=S P_{0} \oplus \triangle S P$ and $S P \hookrightarrow S P^{\prime}\left[\Sigma_{r}\right]$ (resp. $\left.S P \leadsto S P^{\prime}\left[\Sigma_{r}\right]\right)$ but $S P_{0} \nrightarrow S P^{\prime}\left[\Sigma_{r} \cap \Sigma_{0}\right]$ (resp. $S P_{0} \Varangle_{\rightarrow} S P^{\prime}\left[\Sigma_{r} \cap \Sigma_{0}\right]$ ).

Counter-example : Consider the specifications (here $S P_{0}$ is the specification BOOL): 

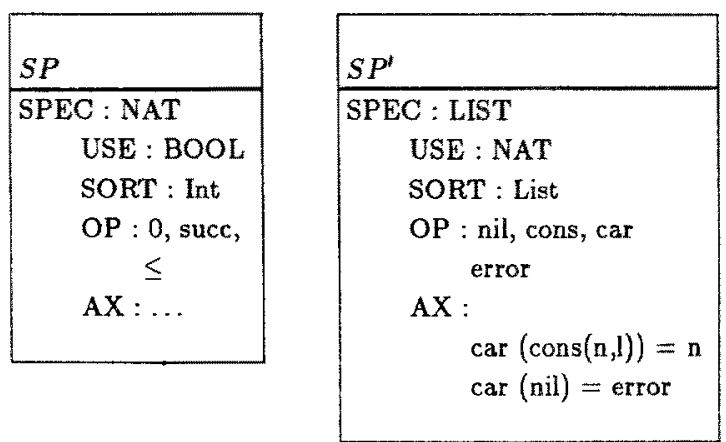

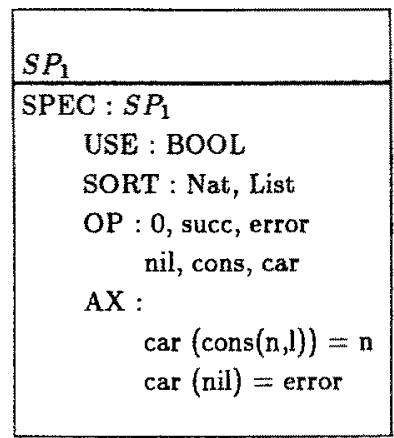

$S P$ is reusable for $S P^{\prime}$ w.r.t. $\Sigma_{N A T}$, but $S P_{0}$ is not reusable for $S P^{\prime}$ w.r.t. $\Sigma_{B O O L}$ via $S P_{1}$. For the $\Sigma_{1}$-algebra $A_{1}$ such that $I n t^{A_{1}}=N \cup$ \{error and (error $\left.\leq n\right)=$ true is a hierarchical model of $S P_{1}$. But $A_{1}$ is not a hierarchical model of $S P^{\prime}$ since $U^{\prime}\left(A_{1}\right)$ is not finitely generated with respect to $\Sigma_{N A T}$ (cf. ertor).

This result seems surprising : if $S P$ is reusable for $S P^{\prime}$, it seems tempting to reuse a primitive part of $S P$ for $S P^{\prime}$. It is well known that as soon as we consider hierarchical specifications, hierarchy is of first importance. This result exemplifies this importance : one may not modify the hierarchy, or ignore it, without care.

It is clear that, if it is possible to flatten the specifications without modification of the hierarchical models, there is no problem. However, fact 5.1 points out that reusability is not generally transitive. Fortunately we will see in part 5.2 that reuse is nevertheless compatible with hierarchy.

Our second result is a kind of symmetric one :

Theorem 5.2 If $S P \hookrightarrow S P^{\prime}\left[\Sigma_{r}\right]$ (resp. $\left.S P \leadsto S P^{\prime}\left[\Sigma_{r}\right]\right)$ and $S P^{\prime}=S P_{0}^{\prime} \oplus \Delta S P^{\prime}$, then $S P \hookrightarrow$ $S P_{0}^{\prime}\left[\Sigma_{\mathbf{r}} \cap \Sigma_{0}^{\prime}\right]$ (resp. $\left.S P \leadsto S P_{0}^{\prime}\left[\Sigma_{\mathbf{r}} \cap \Sigma_{0}^{\prime}\right]\right)$.

Proof : straightforward

\subsection{What about enrichment (efficient case)}

Let us consider now the case when $S P$ is efficiently reusable for $S P^{\prime}$ via $S P_{1}$. What can be done for implementing $S P^{\prime \prime}=S P^{\prime} \oplus \Delta S P^{\prime \prime}$.

Theorem 5.3 Reusability of enrichment.

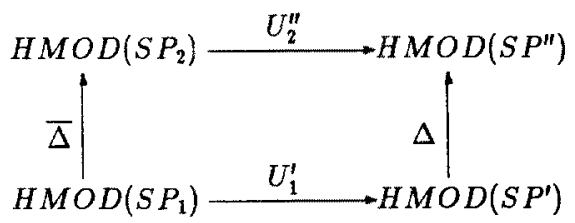

Let $S P, S P_{1}$ and $S P^{\prime}$ be specifications such that $S P \longrightarrow S P_{1} \searrow^{U} S P^{\prime}(S P$ is efficiently reusable for $S P^{\prime}$ w.r.t. some $\Sigma_{r}^{\prime}$ ), and $S P^{\prime \prime}$ a specification which use $S P^{\prime}: S P^{\prime \prime}=S P^{\prime} \oplus \triangle S P^{\prime \prime}$.

If $\Sigma_{1} \cap \Delta \Sigma^{\prime \prime}=\emptyset$, then $S P_{2}=S P_{1} \oplus \Delta S P^{\prime \prime}$ and for all realization $\Delta$ of $\Delta S P^{\prime \prime}$ on top of $S P^{\prime}$, there exists a realization $\bar{\Delta}$ of $\triangle S P^{\prime \prime}$ on top of $S P_{1}$, such that the above diagram commute, i.e. :

$$
\forall A_{1} \in H M O D\left(S P_{1}\right), U_{2}^{\prime \prime} \circ \bar{\Delta}\left(A_{1}\right) \simeq \Delta \circ U_{1}^{\prime}\left(A_{1}\right)
$$


5.1 :

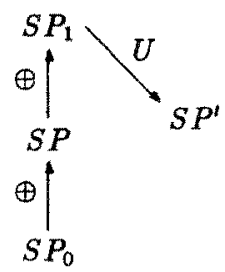

$5.2:$

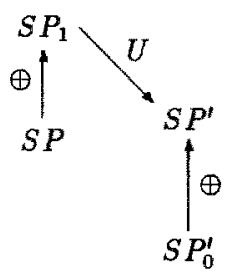

$5.3:$

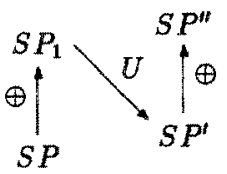

Figure 3: Situations of fact 5.1 and theorems 5.2 and 5.3

\section{Proof : See [GM 87].}

As noted by a referee, this theorem is a generalization of the extension lemma given in [EM 85] for equational specifications and total, initial algebras.

Figure 3 shows the difference between the situations studied in fact 5.1 and theorem 5.3. By the way theorem 5.3 deals with another kind of reusability : those of the realization of $\Delta S P^{\prime \prime}$. Moreover it shows that $\triangle S P^{\prime \prime}$ can be realized independently of the reuse done for $S P^{\prime}$.

The proof of the theorem 5.3 is constructive : it shows how to get a realization of $\Delta S P^{\prime \prime}$ on the top of $S P_{1}$ given a realization of $\triangle S P^{\prime \prime}$ on the top of $S P^{\prime}:$ just putting together the carriers and the operations of $A_{1}$ (which is an implementation of $S P_{1}$ ) and of $A^{\prime \prime}$. Practically it corresponds to putting together type declarations and code of operations.

This result is important : it allows to consider a program as a set of pieces, the bodies of which can be replaced or developed in an independent way. For instance, let us suppose that we have to develop the components below :
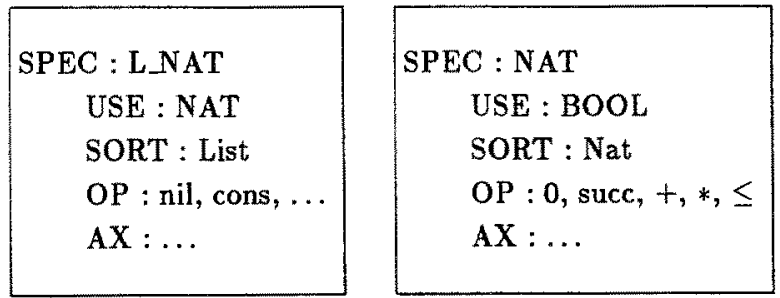

and we already have programs implementing :
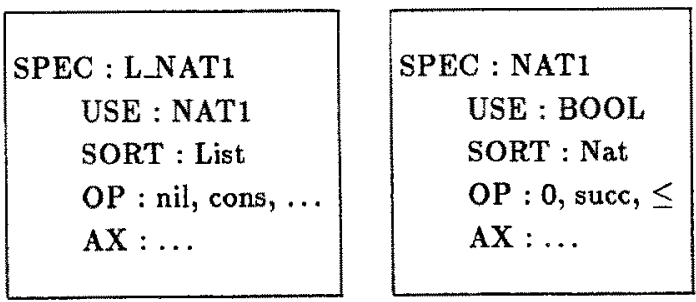

$$
\begin{aligned}
& \text { SPEC : NAT2 } \\
& \text { USE }: \text { BOOL } \\
& \text { SORT }: \text { Nat } \\
& \text { OP }: 0, \text { succ, },+, *, \leq \\
& \text { AX }: \ldots
\end{aligned}
$$

We know, from theorem 5.3 , that it is possible to replace the NAT1 component by the NAT2 component in L_NAT1, and to use L_NAT1 (with NAT2) to implement L_NAT. This avoids to implement addition and multiplication on naturals.

Remark. The condition $\Sigma_{1} \cap \Delta \Sigma^{\prime \prime}=\emptyset$ is essential; it is not a problem to satisfy it using appropriate renaming of what is forgotten from $S P_{1}$ to $S P^{\prime}$. 

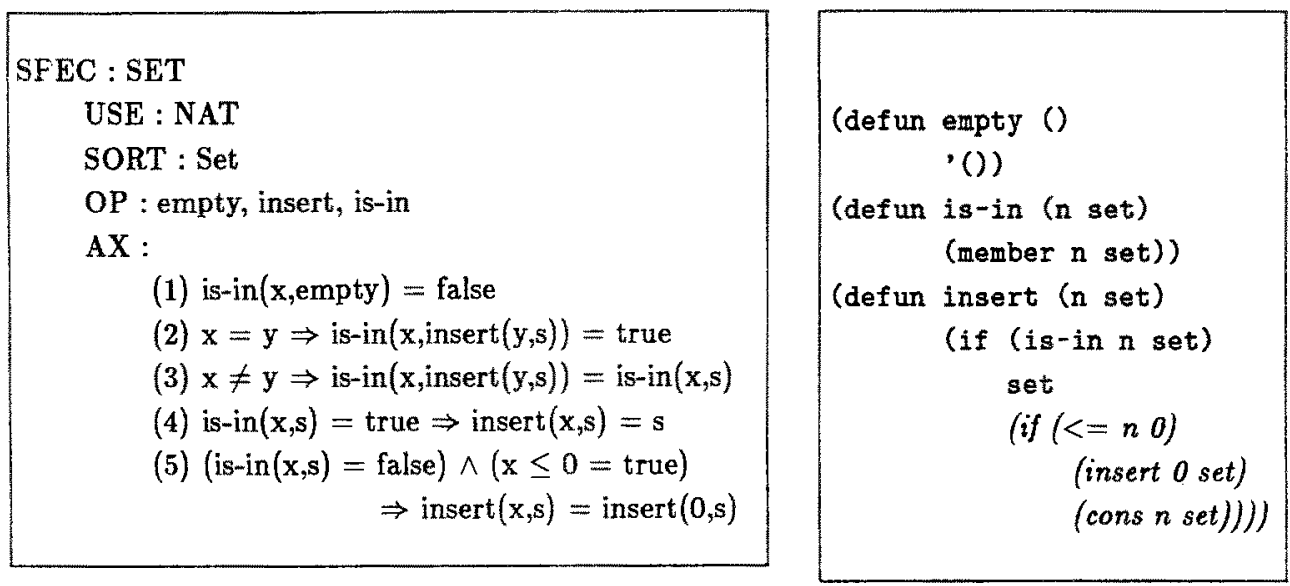

Figure 4: A specification and a (strange) realization of sets of naturals numbers.

\subsection{What about enrichment (non efficient case)}

It would be nice to extend the previous results to the non efficient reusability, unfortunately the previous theorem is no more valid. For instance, let us consider the specifications NAT and INT of figure 1 and the specification SET of the figure 4 (the last axiom looks strange, but it is on purpose).

INT is not efficiently reusable for NAT; SET $=\mathrm{NAT} \oplus \Delta \mathrm{SET}$, but INT $+\Delta \mathrm{SET}$ has no hierarchical models since:

$$
\begin{array}{rlrl}
\text { true } & =\text { is-in(-1,insert }(-1, \text { empty })) & & \text { from (2) } \\
& =\text { is-in(-1,insert(0,empty)) } & \text { from (5) } \\
& =\text { is-in(-1,empty)) } & & \text { from (3) } \\
& =\text { false } & & \text { from (1) }
\end{array}
$$

By the way, the problem is more fundamental than it seems : suppose now that the strange axiom (5) is removed. The LISP program of figure 4 is a correct (but strange) realization of sets of natural numbers. However, it is no more the case if naturals are replaced by relatives. Then, the only way to get such a realization is to define a predicate is-a-nat and to add a check of this predicate in front of each function. Unfortunately, the introduction of such predicates (i.e. subsorts) makes the reuse process much more complicated.

Definition 5.1 Let $S P_{0} \subseteq S P$ two specifications which use a specification $B O O L$ of booleans. $A$ discriminant predicate between $S P$ and $S P_{0}$, for $s \in S_{0}$, is an operation $\left(p_{s}: s \longrightarrow B o o l\right) \in F$ such that :

$$
\forall A \in P G E N(S P), \forall a \in s^{A}, p_{s}^{A}(a)= \begin{cases}\text { true } & \text { if } a \in s^{V(A)} \\ \text { false } & \text { otherwise }\end{cases}
$$

If $s \in S \backslash S_{0}$, by convention, $p_{s}^{A}(a)=f a l s e, \forall a \in s^{A}$. 


\section{Remark.}

A discriminant predicate is the characteristic function of the finitely generated part of $s^{V(A)}$. If SP and $S P_{0}$ have free generators $C$ and $C_{0}\left(C_{0} \subseteq C\right)$, the definition of the $p_{s}$ predicates is straightforward :

- $p_{s}\left(c_{0}\right)=t r u e$ for all constant $\left(c_{0}: \longrightarrow s\right) \in C_{0}$.

$-p_{s_{1}}\left(x_{1}\right)=\operatorname{true} \wedge \ldots \wedge p_{s_{n}}\left(x_{n}\right)=$ true $\Longleftrightarrow p_{s}\left(f_{0}\left(x_{1} \ldots x_{n}\right)\right)=$ true for all $\left(f_{0}: s_{1} \ldots s_{n} \longrightarrow\right.$ $s) \in C_{0}$.

- $p_{s}(c)=$ false for all constant $(c:-\rightarrow s) \in C \backslash C_{0}$.

- $p_{s}\left(f\left(x_{1} \ldots x_{n}\right)\right)=$ false for all $\left(f: s_{1} \ldots s_{n} \longrightarrow s\right) \in C \backslash C_{0}$.

If it is not the case, the definition of these predicates is highly dependent on the rest of the specification.

Definition 5.2 : extended enrichment.

Let $S P_{0} \subseteq S P_{1}$ be two specifications, $\triangle S P$ an enrichment of $S P_{0}$, such that $\Sigma_{1} \cap \Delta \Sigma=0$, and $\left(p_{s}\right)_{s \in S_{1}}$ the discrimant predicates between $S P_{0}$ and $S P_{1}$.

An extended enrichment of $\triangle S P$ from $S P_{0}$ to $S P_{1}$, is the enrichment $\overline{\Delta S P}=\left(\Delta \Sigma, \widehat{\Delta E} \cup P_{\Delta \Sigma}\right)$ where :

- $P_{\Delta \Sigma}$ is the set of axioms : $D\left(f\left(x_{1}, \ldots, x_{i}, \ldots, x_{n}\right)\right) \Longrightarrow p_{s_{i}}\left(x_{i}\right)=$ true, where $\left(f: s_{1} \ldots s_{n} \longrightarrow\right.$ s) $\in \Delta F$ and $s_{i} \in S_{0}$.

- $\widehat{\Delta E}$ is obtained from $\Delta E$ by the following transformations :

- If an axiom is of the form : $D\left(\theta_{1}\right) \wedge \ldots \wedge D\left(\theta_{m}\right) \wedge\left(t_{1}=t_{1}^{\prime}\right) \wedge \ldots \wedge\left(t_{n}=t_{n}^{\prime}\right) \Longrightarrow\left(t=t^{\prime}\right)$, it becomes : $\Phi \wedge D\left(\theta_{1}\right) \wedge \ldots \wedge D\left(\theta_{m}\right) \wedge\left(t_{1}=t_{1}^{\prime}\right) \wedge \ldots \wedge\left(t_{n}=t_{n}^{\prime}\right) \Longrightarrow\left(t=t^{\prime}\right)$ where : $\Phi=\Lambda_{i}\left[p_{s_{i}}\left(\theta_{i}\right)=\right.$ true $] \wedge \Lambda_{j}\left[p_{s_{j}^{\prime}}\left(t_{j}\right)=\right.$ true $\wedge p_{s_{j}^{\prime}}\left(t_{j}^{\prime}\right)=$ true $] \wedge\left[p_{s}(t)=\right.$ true $\wedge p_{s}\left(t^{\prime}\right)=$ true $]$ ( $s_{i}$ is the sort of $\theta_{i}, s_{j}^{\prime}$ is the sort of $t_{j}$ et $t_{j}^{\prime}, s$ is the sort of $t$ and $t^{\prime}$. Moreover, in order to simplify the transformation, we define, for $s \in \Delta S, p_{s}(x)=$ true, $\forall x$; these predicates are useless but for the transformation.)

- If an axiom is of the form :

$D\left(\theta_{1}\right) \wedge \ldots \wedge D\left(\theta_{m}\right) \wedge\left(t_{1}=t_{1}^{\prime}\right) \wedge \ldots \wedge\left(t_{n}=t_{n}^{\prime}\right) \Longrightarrow D\left(f\left(t_{1}^{\prime \prime}, \ldots, t_{p}^{\prime \prime}\right)\right)$, it becomes :

$\Phi \wedge D\left(\theta_{1}\right) \wedge \ldots \wedge D\left(\theta_{m}\right) \wedge\left(t_{1}=t_{1}^{\prime}\right) \wedge \ldots \wedge\left(t_{n}=t_{n}^{\prime}\right) \Rightarrow D\left(f\left(t_{1}^{\prime \prime}, \ldots, t_{p}^{\prime \prime}\right)\right)$ where:

$\Phi=\Lambda_{i}\left[p_{s_{i}}\left(\theta_{i}\right)=\right.$ true $] \wedge \Lambda_{j}\left[p_{s_{j}^{\prime}}\left(t_{j}\right)=\right.$ true $\wedge p_{s_{j}^{\prime}}\left(t_{j}^{\prime}\right)=$ true $] \wedge \wedge_{k}\left[p_{s_{k}^{\prime \prime}}\left(t_{k}^{\prime \prime}\right)=\right.$ true $]$

(with the same conventions as above, and $s_{k}^{\prime \prime}$ is the sort of $t_{k}^{\prime \prime}$ )

The $P_{\Delta \Sigma}$ axioms express that the operations of $\Delta S P$, when $S P_{1}$ is used instead of $S P_{0}$, must be restricted to the parts of the sorts of $S P_{1}$ which are finitely generated by $\Sigma_{0}$. Similarly, the $\Phi$ premisses added to the axioms $\Delta E$ express that these axioms are valid on these parts of the sorts. Examples of this transformation can be found in [GM 87].

We have a theorem similar to theorem 5.3 :

Theorem 5.4 Reusability of enrichment.

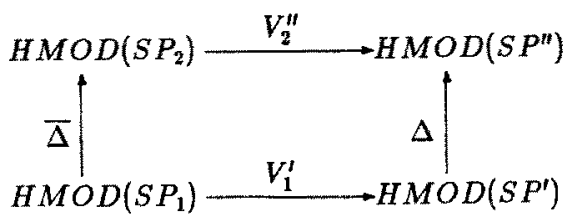


Let $S P, S P_{1}$ et $S P^{\prime}$ be specifications such that $S P \longrightarrow S P_{1} \searrow^{V} S P^{\prime}\left(S P\right.$ is reusable for $S P^{\prime}$ w.r.t. some $\left.\Sigma_{r}^{\prime}\right)$, and $S P^{\prime \prime}=S P^{\prime} \oplus \Delta S P^{\prime \prime}$, with $\Sigma_{1} \cap \Delta \Sigma^{\prime \prime}=\emptyset$.

Then $S P_{2}=S P_{1} \oplus \overline{\Delta S P^{\prime \prime}}$, and for all realization $\Delta \in R E A L_{S P^{\prime}}\left(\triangle S P^{\prime \prime}\right)$, there is a realization $\bar{\Delta} \in R E A L_{S P_{1}}(\overline{\triangle S P \prime})$, such that the diagram above commutes, i.e. :

$$
\forall A_{1} \in H M O D\left(S P_{1}\right), V_{2}^{\prime \prime} \circ \bar{\Delta}\left(A_{1}\right) \simeq \Delta \circ V_{1}^{\prime}\left(A_{1}\right)
$$

Proof: See [GM 87].

\section{Conclusions and further researches}

We have given a criterion for "efficient" software reusability, and proved that this notion of reusability fits well for hierarchical specifications. We have introduced "non efficient reusability" (such as integer for naturals) in order to be more permissive. However, the results on this kind of reusability are slightly disappointing since they seem rather complex to apply.

It is interesting to note that non efficient reusability is not exactly what we have called "direct reusability", and is a step forward implementation. These results enforce our opinion that the kind of reusability we are studying, i.e. code reusability using its specification, should be done on as-it-is bases whenever possible. This is quite coherent with practice.

This study was devoted to structured specification and modular programs. It is clear that it must be extended to parameterized specifications and generic programs.

\section{Acknowledgements}

Our thanks to Michel Bidoit and Gilles Bernot for numerous fruitful discussions and friendly encouragements.

This work is partially funded by ESPRIT (Meteor Project) and by the PRC-Greco "Programmation et outils pour l'intelligence artificielle". Th. Moineau's grant is funded by Sema-Metra.

\section{References}

[BBC 86] G. Bernot, M. Bidoit and C. Choppy, "Abstract implementation and correctness proofs", in Proc. 3rd STACS, Jan. 1986, Springer-Verlag LNCS 210, Jan. 1986.

[Ber 87] G. Bernot, "Good functors ... are those preserving philosophy !", in Proc. 2nd Summer Conference on Cotegory Theory and Computer Science, Edinburgh, Sept. 1987.

also LRI report No. 354, June 1987.

[Bid 87] M. Bidoit, "The stratified loose approach : A generalization of initial and loose semantics", to appear.

[BW 82] M. Broy and M. Wirsing, "Partial abstract types", Acta Informatica, No. 18, 1982.

[EKMP 80] H. Ehrig, H. Kreowski, B. Mahr and P. Padawitz, "Algebraic implementation of abstract data types", Theoretical Computer Science, Oct. 1980. 
[EM 85] H. Ehrig and B. Mahr, "Fundamentals of algebraic specification", Springer Verlag, Berlin-Heidelberg-New York-Tokyo, 1985.

[FGJM 85] K. Futatsugi, J.A. Goguen, J-P. Jouannaud and J. Meseguer, "Principles of OBJ2", in proc. 12th ACM Symposium on Principles of Programming Languages, Jan. 1985.

[Gau 85] M.C. Gaudel, "Towards structured algebraic specifications", ESPRIT'85 - Status Report, Part I, pp, 493-510, North Holland, 1986.

[GM 87] M.C. Gaudel and Th. Moineau, "A theory of software reusability", LRI report No. 380 , Oct. 1987.

|GH 78] J.V. Guttag and J.J. Horning, "The algebraic specification of abstract data types." Acta Informatica, No. 10, 1978.

[Pro 82] K. Proch, "ORSEC : Un Outil de Recherche de Spécifications Equivalentes par Comparaison d'exemple", Thèse de 3eme cycle, Nancy I, Dec. 1982.

[SW 82] D. Sanella and M. Wirsing, "Implementation of parametrized specifications", Report CSR-102-82, Department of Computer Science, University of Edinburgh.

[Wir 83] M. Wirsing, "Structured algebraic specifications : a kernel language", Habilitation Thesis, Technische Universität München, Sept. 1983.

[WPPDB 83] M. Wirsing, P. Pepper, H. Partsch, W. Dosch and M. Broy, "On hierarchy of abstract data types", Acta Informatica, No. 20, 1983. 